\title{
Haplo-insufficiency? Let me count the ways
}

\author{
Kim C. Quon and Anton Berns ${ }^{1}$ \\ Division of Molecular Genetics and Centre for Biomedical Genetics, The Netherlands Cancer Institute, 1066 CX \\ Amsterdam, The Netherlands
}

During the course of cancer development, a normal cell progresses toward malignancy by acquiring a specific series of mutations. These include mutations that activate otherwise innocuous proto-oncogenes, and others that inactivate recessive tumor suppressor genes. By acquiring these mutations, a cell progressively alters its phenotype, and thereby eludes the various controls that normally prevent malignant growth in an organism. Based on epidemiological data (Renan 1993), and consistent with in vitro experimental data (Hahn et al. 1999), it is estimated that between four and eight rate-limiting mutations occur during the development of most human cancers. But this raises a conundrum. The incidence of cancer should be proportional to the number of rate-limiting events necessary for tumorigenesis, the frequency of these events, and the size of the target cell population for these events. Therefore, given that somatic mutations arise at a frequency of $<6 \times 10^{-6}$ per locus (Seshadri et al. 1987), an overly simplistic calculation would suggest that even a tumor requiring only four mutations would only arise at a frequency of $\sim 1$ in $10^{-21}$ cells, a vanishingly low frequency even in an organism composed of $\sim 10^{14}$ cells, as humans are. Why, then, are the odds of developing cancer during one's lifetime $\sim 1$ in 3 , and what does this tell us about the mechanisms that operate during tumorigenesis?

At least two factors confound the simplicity of the above calculation. The first concerns the frequency of occurrence of the rate-limiting events. In tumors, genes may be epigenetically silenced by methylation as well as inactivated by genetic mutation (Jones and Laird 1999), and, therefore, the mutation rate does not necessarily accurately reflect the overall rate of gene inactivation. Furthermore, nearly all human tumors show an enhanced mutation rate or chromosomal instability (Lengauer et al. 1998). The total frequency of gene inactivation in tumor cells may therefore be up to 1000-fold higher than in normal cells (Seshadri et al. 1987).

A second confounding factor concerns the size of the target cell population that undergoes mutation. If a single mutation, either activation of an oncogene or in-

${ }^{1}$ Corresponding author.

E-MAIL tberns@nki.nl; FAX 31-20-512-20-11.

Article and publication are at http://www.genesdev.org/cgi/doi/10.1101/ gad.949001. activation of a tumor suppressor gene, were to confer a significant selective advantage on an emerging tumor cell, the resulting clonal expansion would vastly increase the cellular target size for each subsequent mutation, and thus increase the overall frequency of tumorigenesis (e.g., see Fig. 1B). Is the high incidence of cancer in human populations telling us that this second mechanism also must play a significant role in cancer development in vivo? The appearance of characteristic mutations at each increasingly malignant stage of colon carcinoma progression (Fearon and Vogelstein 1990) suggests that selection for intermediate-stage, premalignant genetic lesions does, in fact, occur. However, the precise details of how this occurs remain unclear. For example, although one might expect that mutations that activate oncogenes would confer a selective advantage on a cell, by driving cell cycle progression and/or by inducing genomic instability, the converse appears, paradoxically, to be true as well: in normal cells, overexpression of oncogenes such as Ras, Myc, and E1a activates an intracellular tumor surveillance mechanism (see below) and results in cell cycle arrest or apoptosis rather than clonal expansion (Serrano et al. 1997; de Stanchina et al. 1998; Zindy et al. 1998). It is also not obvious how inactivating mutations in tumor suppressor genes would confer a selective advantage. Although complete loss of a tumor suppressor gene function could provide a selective advantage, the prevailing paradigm for tumor suppressor gene function, Knudson's two-hit model (Knudson 1985), states that tumor suppressor genes are recessive and that both alleles must be inactivated for tumorigenesis. How, then, could the intermediate step, the mutation of a single allele, contribute to clonal expansion?

With respect to this last question, the answer is clear: loss of a single allele of a tumor suppressor gene can confer a selective advantage, as has been expertly reviewed recently (Cook and McCaw 2000). For example, inactivation of a single allele of the gene encoding the cyclin-dependent kinase inhibitor $\mathrm{p} 27^{\mathrm{Kip} 1}$ is sufficient to sensitize mice to radiation and carcinogen-induced tumorigenesis (Fero et al. 1998). The tumors that arise retain expression of an unmutated wild-type allele, showing that loss of a single tumor suppressor gene allele can, indeed, provide a selective advantage during tumorigenesis. In this issue, Inoue et al. (2001) describe another clear example of a gene, $D m p 1$, that is haplo-insufficient for tumor suppression, emphasizing the idea that haplo- 


\section{A. strong haplo-insufficiency}
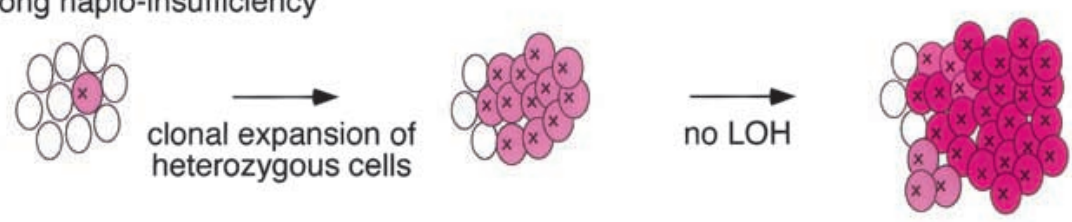

heterozygous

mutant tumor

B. partial haplo-insufficiency

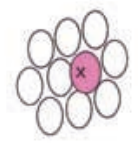

clonal expansion of heterozygous cells
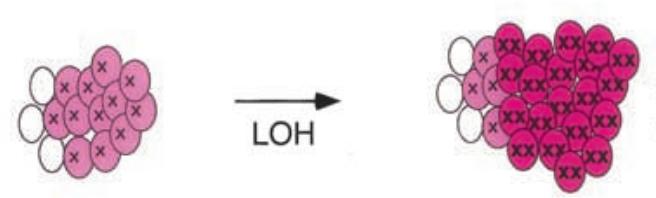

homozygous

mutant tumor

\section{C. no haplo-insufficiency}
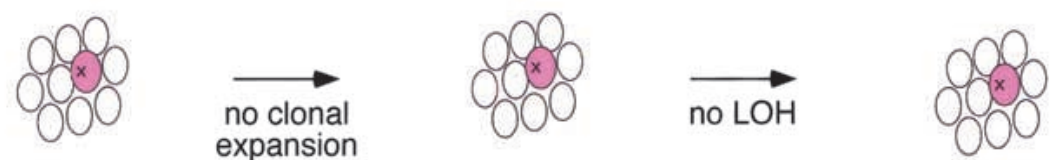

no tumor expansion

\section{D. familial cancer, with or without haplo-insufficiency}
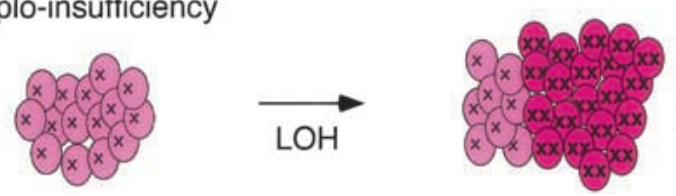

homozygous mutant tumor

Figure 1. Haplo-insufficiency increases the probability of completing a multistep tumorigenesis pathway. Strong $(A)$ or partial $(B)$ haplo-insufficiency allows cells that are heterozygous for a tumor suppressor gene to undergo clonal expansion. This increases the size of the target cell population available to complete a multistep tumorigenesis pathway, and thus greatly increases the probability of tumors occurring. For partially haplo-insufficient tumor suppressor genes $(B)$, loss of the remaining tumor suppressor gene allele (loss of heterozygosity, $\mathrm{LOH}$ ) is required to complete tumorigenesis. $(C)$ In the absence of haplo-insufficiency, no clonal expansion of heterozygous cells occurs, and therefore insufficient numbers of cells are available to complete the pathway, and tumors fail to occur or only rarely occur. $(D)$ In familial cancers, all cells are heterozygous to begin with, and therefore no clonal expansion is necessary to increase the size of heterozygous target cell population. Therefore, tumors can arise even in the absence of haplo-insufficiency. Cells carrying two wild-type alleles of a tumor suppressor gene are shown in white; heterozygous cells with one mutant allele are in light pink; tumor cells with one or two mutant alleles are in dark pink; $(\mathrm{X})$ mutant tumor suppressor alleles.

insufficiency may be a much more widespread feature of tumor suppressor genes than is generally realized.

Cyclin D-binding Myb-like protein (Dmp1) is a transcription factor that was originally isolated as a cyclin D2-interacting protein in a yeast two-hybrid screen (Hirai and Sherr 1996). Although numerous potential nonameric DNA-binding sites for Dmp1 exist in the genome, the ability of Dmp1 to induce cell cycle arrest appears to reside primarily in its ability to transactivate a single target gene, the tumor suppressor gene Arf (Inoue et al. 1999). The product of the Arf gene, p19 ${ }^{\mathrm{Arf}}\left(\mathrm{p} 14^{\mathrm{ARF}}\right.$ in humans), is a component of a signaling pathway thought to act as an intracellular tumor surveillance system that arrests or destroys incipient tumor cells that have undergone inappropriate oncogene activation (Eischen et al. 1999). Upon induction, p19 ${ }^{\text {Arf }}$ inactivates the p53 antagonist $\mathrm{Mdm} 2$, thereby stabilizing and activating p53 and inducing cell cycle arrest, cellular senescence, or apoptosis, depending on the cellular context. Mutations in the human versions of $A r f, M d m 2$ (HDM2 in humans), and p53 are found in the vast majority of human tumors, underscoring the importance of this pathway in tumor suppression. Interestingly, Dmp1's ability to induce the p19 ${ }^{\text {Arf }}-$ Mdm2-p53 signaling pathway is inhibited by its interaction with D-type cyclins (Inoue and Sherr 1998). Because D cyclins are rapidly induced by growth factors during the initiation of the cell cycle (Matsushime et al. 1991), Dmp1 sequestration by cyclin D may be a mechanism that allows cells to inactivate or attenuate p19 $9^{\text {Arf }}$ Mdm2-p53-mediated cell cycle arrest during growth factor-induced proliferation. Although the physiological significance of the cyclin $\mathrm{D} / \mathrm{Dmp} 1$ interaction has yet to be established, this raises the possibility that cyclin D and Dmpl may be part of a mechanism that distinguishes desired, growth factor-induced cell cycle progression from the undesired, potentially oncogenic proliferation induced by oncogenes such as Ras, Myc, and E1a.

Mouse embryo fibroblasts (MEFs) lacking Dmp1 show reduced expression of $\mathrm{p} 19^{\mathrm{Arf}}$ and $\mathrm{p} 53$, manifest a greatly prolonged proliferative capacity in culture, and can be 
transformed by oncogenic Ras (Inoue et al. 2000), suggesting that Dmp1 indeed acts as a cellular modulator of the p19 ${ }^{\text {Arf }}-M d m 2-p 53$ tumor suppressor pathway. Inoue et al. (2001) now show that Dmp1 is a bona fide tumor suppressor gene: Dmp1 nullizygous mice spontaneously develop a wide variety of lethal tumors during their second year of life, and show dramatically accelerated lymphomagenesis in the presence of an oncogenic $\mathrm{E} \mu-M y c$ transgene. Surprisingly, T-cell lymphomas, lung tumors, and $\mathrm{E} \mu-M y c$-induced B-cell lymphomas arose with similar latencies in either Dmp1 hemizygous or Dmp1 nullizygous mice. The wild-type Dmp1 allele was retained and expressed in the vast majority of tumors arising from Dmp1 hemizygous mice, and in 5/5 tumors examined, the wild-type protein was shown to be functional in specific DNA-binding assays and to contain no alterations within the DNA-binding domain, which strongly supports the argument that, at least for this subset of tumor types, Dmp1 is haplo-insufficient for tumor suppression in mice.

Importantly, Dmp1 inactivation (either hemi- or nullizygous) appears to reduce the requirement for inactivation of the p19 $9^{\text {Arf }}-\mathrm{Mdm} 2-\mathrm{p} 53$ pathway during E $\mathrm{L}-\mathrm{Myc}$ induced lymphomagenesis. Whereas in an $\mathrm{E} \mu-M y c$ $D m p 1^{+/+}$background, $\sim 50 \%$ of tumors show mutational inactivation of Arf or p53, in a Dmp1 mutant background, only 9\% (Eu-Myc Dmp $\left.1^{-/-}\right)$or $14 \%(\mathrm{E} \mu-M y c$ $D m p 1^{+/-}$do. This, and the fact Dmp1 loss appears to phenocopy Arf hemizygosity in accelerating tumorigenesis in this system, together provide a strong argument that $D m p 1$ acts as a tumor suppressor in vivo at least in part by modulating p19 Arf expression. As the authors suggest, this raises the possibility that other components of the p19 Arf $-M d m 2-$ p53 signaling pathway may also show haplo-insufficiency under some circumstances. This, in fact, appears to be the case: p53 hemizygous mice give rise to tumors that retain and express functional wild-type p53 at a frequency of $>50 \%$ (Venkatachalam et al. 1998). Moreover, in the absence of a functional $p 16^{\operatorname{Ink} 4 a}$ tumor suppressor gene, loss of a single Arf allele appears to be sufficient for tumorigenesis (Krimpenfort et al. 2001). Interestingly, 50\% (4/8) of $D \mathrm{mp} 1^{+/-}$lung tumors examined by Inoue et al. showed methylation of the $p 16^{\operatorname{Ink} 4 a}$ promoter, suggesting that like Arf hemizygosity, Dmp1 hemizygosity also collaborates with $p 16^{\text {Ink } 4 a}$ inactivation to promote tumorigenesis.

Notably, whereas $D m p 1$ haplo-insufficiency is most apparent in the presence of an $\mathrm{E} \mu-M y c$ transgene, in this background, Arf is clearly not haplo-insufficient (Eischen et al. 1999). This suggests that Dmpl may act through genes in addition to Arf to suppress tumorigenesis, a notion that is further supported by the fact that strong overexpression of Dmp1 is capable of inducing cell cycle arrest even in NIH 3T3 cells that do not express p19 Arf (Inoue and Sherr 1998). In this regard, it would of great interest to identify additional relevant transcriptional targets of Dmp1.

With these latest results, Dmp1 joins $p 27^{\text {Kip1 }}$ as another example of a haplo-insufficient tumor suppressor gene that does not conform to Knudson's two-hit model of tumor suppressors. However, even though both genes are clearly haplo-insufficient for tumor suppression, both $p 27^{\text {Kip } 1}$ and Dmp1 mutant mice show at least slightly more severe tumor susceptibility phenotypes in the homozygous state than in the heterozygous state for most tumor types. This, along with the fact that there are clearly examples of tumor suppressor genes that show haplo-insufficiency only in the presence of specific additional mutations $(A r f)$, or that show weaker haploinsufficiency (p53), suggests that haplo-insufficiency for tumor suppression is not a black-and-white situation. Instead, it argues that tumor suppressor genes show varying degrees of haplo-insufficiency, with some genes showing strong effects, some showing partial effects, and others showing little or none (Cook and McCaw 2000).

If we include weaker, partial effects, how widespread is haplo-insufficiency among tumor suppressor genes? As alluded to above, haplo-insufficiency, however weak, would allow the clonal expansion of cells carrying a single mutated copy of a tumor suppressor gene, and would thus increase the size of the target cell population available for subsequent mutations during the remaining course of tumor progression (Fig. 1B). Thus, just as genomic instability appears to be required to generate a sufficiently high mutation rate for tumorigenesis, some degree of haplo-insufficiency may be required to generate a sufficiently large target cell population for mutagenesis (including mutation or loss of the remaining tumor suppressor gene allele). Therefore, despite the fact that only a handful of haplo-insufficient tumor suppressor genes have been definitively identified, haplo-insufficiency, in some form or another, may be a hallmark of essentially all tumor suppressor genes that are frequently mutated in sporadic human cancers.

An important corollary to this hypothesis is that tumor suppressor genes implicated solely in familial cancers are not necessarily haplo-insufficient. Patients with familial cancer susceptibility syndromes that conform to Knudson's two-hit model are cancer-prone because they inherit a single mutated tumor suppressor gene allele that is present in every cell of their body (Knudson 1985), and therefore clonal expansion of the premalignant heterozygous mutant cells does not necessarily play a role during tumor progression in these individuals (Fig. 1D). Most of the inherited cancer susceptibility genes identified to date are also found to be mutated in sporadic human cancers and therefore would be expected to be haplo-insufficient. However, two noteworthy exceptions are the BRCA1 and BRCA2 genes, which confer susceptibility to breast and ovarian cancer when mutated in the germ line, but which have not, or have only rarely been found to be mutated in sporadic tumors (Merajver et al. 1995; Lancaster et al. 1996; Couch and Weber 1998). Intriguingly, unlike homozgyous mutant BRCA2 MEFs, heterozygous BRCA2 MEFS do not show detectable defects in DNA repair in vitro (Patel et al. 1998) This lack of haplo-insufficiency may also apply to $B R C A 1$, but to the best of our knowledge this has yet to be documented. Although it remains to be proven that DNA repair is the 
relevant biochemical function for tumor suppression by these genes in vivo, we would argue that whatever the relevant biochemical activity, it is the inability of heterozygous $B R C A 1$ and $B R C A 2$ mutant cells to undergo clonal expansion in vivo that underlies the notable paucity of mutations in these genes in sporadic human cancer.

Is there experimental data to support the contention that haplo-insufficiency is a widespread phenomenon among tumor suppressor genes? Although most of the evidence to date for haplo-insufficiency, weak or strong, comes from mouse knockout studies, the results from human mutational analyses are consistent with this possibility. In collections of tumors with hemizygous deletions at a given tumor suppressor locus, loss of the remaining tumor suppressor gene allele is seldom detected in all of the tumors. The absence of detectable mutations in a subset of the tumors has usually been attributed to inactivation of the remaining allele in this subset by other mechanisms such as epigenetic silencing, mutations in noncoding regions, or by the presence of other unidentified tumor suppressor genes within the region of deletion. However, this failure may actually be reflecting, at least in part, weak haplo-insufficiency.

Strongly haplo-insufficient tumor suppressor genes like Dmp1 do not conform to Knudson's two-hit model, because the model defines a tumor suppressor gene based on the presence of mutations or deletions in both alleles of a gene in tumors. Thus, the list of human tumor suppressor genes identified to date is necessarily skewed toward genes that show weak or no haplo-insufficiency. If this hypothesis is correct, that haplo-insufficiency for tumor suppression is much more widespread than previously appreciated, this definition may be too stringent. Indeed, the numerous regions of loss of heterozygosity (LOH) identified in human cancer for which no tumor suppressor gene has been identified-including 7q21, where human DMP1 resides (Bodner et al. 1999)—may be harboring a treasure trove of haplo-insufficient tumor suppressor genes that will never be found by traditional mutational analysis or criteria.

\section{Acknowledgments}

We thank Maarten van Lohuizen, Paul Krimpenfort, and Jos Jonkers for critical reading of the manuscript.

\section{References}

Bodner, S.M., Naeve, C.W., Rakestraw, K.M., Jones, B.G., Valentine, V.A., Valentine, M.B., Luthardt, F.W., Willman, C.L., Raimondi, S.C., Downing, J.R., et al. 1999. Cloning and chromosomal localization of the gene encoding human cyclin D-binding Myb-like protein (hDMP1). Gene 229: 223228.

Cook, W.D. and McCaw, B.J. 2000. Accommodating haploinsufficient tumor suppressor genes in Knudson's model. Oncogene 19: 3434-3438.

Couch, F. and Weber, B. 1998. Breast cancer. In The genetic basis of cancer (eds. B. Vogelstein and K.W. Kinzler), pp. 537-564. McGraw Hill, New York, NY.

de Stanchina, E., McCurrach, M.E., Zindy, F., Shieh, S.Y., Ferbeyre, G., Samuelson, A.V., Prives, C., Roussel, M.F., Sherr, C.J., and Lowe, S.W. 1998. E1A signaling to p53 involves the p19(ARF) tumor suppressor. Genes \& Dev. 12: 2434-2442.

Eischen, C.M., Weber, J.D., Roussel, M.F., Sherr, C.J., and Cleveland, J.L. 1999. Disruption of the Arf-Mdm2-p53 tumor suppressor pathway in Myc-induced lymphomagenesis. Genes \& Dev. 13: 2658-2669.

Fearon, E.R. and Vogelstein, B. 1990. A genetic model for colorectal tumorigenesis. Cell 61: 759-767.

Fero, M.L., Randel, E., Gurley, K.E., Roberts, J.M., and Kemp, C.J. 1998. The murine gene p27Kip1 is haplo-insufficient for tumour suppression. Nature 396: 177-180.

Hahn, W.C., Counter, C.M., Lundberg, A.S., Beijersbergen, R.L., Brooks, M.W., and Weinberg, R.A. 1999. Creation of human tumour cells with defined genetic elements. Nature 400: 464-468.

Hirai, H. and Sherr, C.J. 1996. Interaction of D-type cyclins with a novel myb-like transcription factor, DMP1. Mol. Cell. Biol. 16: 6457-6467.

Inoue, K. and Sherr, C.J. 1998. Gene expression and cell cycle arrest mediated by transcription factor DMP1 is antagonized by D-type cyclins through a cyclin-dependent-kinase-independent mechanism. Mol. Cell. Biol. 18: 15901600.

Inoue, K., Roussel, M.F., and Sherr, C.J. 1999. Induction of ARF tumor suppressor gene expression and cell cycle arrest by transcription factor DMP1. Proc. Nat1. Acad. Sci. 96: 39933998.

Inoue, K., Wen, R., Rehg, J.E., Adachi, M., Cleveland, J.L., Roussel, M.F., and Sherr, C.J. 2000. Disruption of the ARF transcriptional activator DMP1 facilitates cell immortalization, Ras transformation, and tumorigenesis. Genes \& Dev. 14: 1797-1809.

Inoue, K., Zindy, F., Randle, D.H., Rehg, J.E., and Sherr, C.J. 2001. Dmp1 is haplo-insufficient for tumor suppression and modifies the frequencies of Arf and p53 mutations in Mycinduced lymphomas. Genes \& Dev. 15: 2934-2939 (this issue).

Jones, P.A. and Laird, P.W. 1999. Cancer epigenetics comes of age. Nat. Genet. 21: 163-167.

Knudson, Jr., A.G. 1985. Hereditary cancer, oncogenes, and antioncogenes. Cancer Res. 45: 1437-1443.

Krimpenfort, P., Quon, K.C., Mooi, W.J., Loonstra, A., and Berns, A. 2001. Loss of p16Ink4a confers susceptibility to metastatic melanoma in mice. Nature 413: 83-86.

Lancaster, J.M., Wooster, R., Mangion, J., Phelan, C.M., Cochran, C., Gumbs, C., Seal, S., Barfoot, R., Collins, N., Bignell, G., et al. 1996. BRCA2 mutations in primary breast and ovarian cancers. Nat. Genet. 13: 238-240.

Lengauer, C., Kinzler, K.W., and Vogelstein, B. 1998. Genetic instabilities in human cancers. Nature 396: 643-649.

Matsushime, H., Roussel, M.F., Ashmun, R.A., and Sherr, C.J. 1991. Colony-stimulating factor 1 regulates novel cyclins during the $\mathrm{G}_{1}$ phase of the cell cycle. Cell 65: 701-713.

Merajver, S.D., Pham, T.M., Caduff, R.F., Chen, M., Poy, E.L., Cooney, K.A., Weber, B.L., Collins, F.S., Johnston, C., and Frank, T.S. 1995. Somatic mutations in the BRCA1 gene in sporadic ovarian tumours. Nat. Genet. 9: 439-443.

Patel, K.J., Yu, V.P., Lee, H., Corcoran, A., Thistlethwaite, F.C., Evans, M.J., Colledge, W.H., Friedman, L.S., Ponder, B.A., and Venkitaraman, A.R. 1998. Involvement of Brca2 in DNA repair. Mol. Cell 1: 347-357. 
Renan, M.J. 1993. How many mutations are required for tumorigenesis? Implications from human cancer data. Mol. Carcinog. 7: 139-146.

Serrano, M., Lin, A.W., McCurrach, M.E., Beach, D., and Lowe, S.W. 1997. Oncogenic ras provokes premature cell senescence associated with accumulation of p53 and p16INK4a. Cell 88: 593-602.

Seshadri, R., Kutlaca, R.J., Trainor, K., Matthews, C., and Morley, A.A. 1987. Mutation rate of normal and malignant human lymphocytes. Cancer Res. 47: 407-409.

Venkatachalam, S., Shi, Y.P., Jones, S.N., Vogel, H., Bradley, A., Pinkel, D., and Donehower, L.A. 1998. Retention of wildtype p53 in tumors from p53 heterozygous mice: Reduction of p53 dosage can promote cancer formation. EMBO $\mathrm{T}$. 17: 4657-4667.

Zindy, F., Eischen, C.M., Randle, D.H., Kamijo, T., Cleveland, J.L., Sherr, C.J., and Roussel, M.F. 1998. Myc signaling via the ARF tumor suppressor regulates p53-dependent apoptosis and immortalization. Genes \& Dev. 12: 2424 2433. 


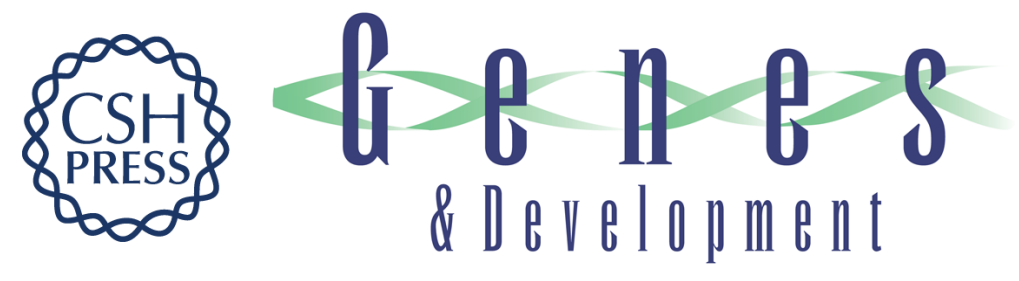

\section{Haplo-insufficiency? Let me count the ways}

Kim C. Quon and Anton Berns

Genes Dev. 2001, 15:

Access the most recent version at doi:10.1101/gad.949001

References This article cites 25 articles, 11 of which can be accessed free at: http://genesdev.cshlp.org/content/15/22/2917.full.html\#ref-list-1

License

Email Alerting Receive free email alerts when new articles cite this article - sign up in the box at the top Service right corner of the article or click here.

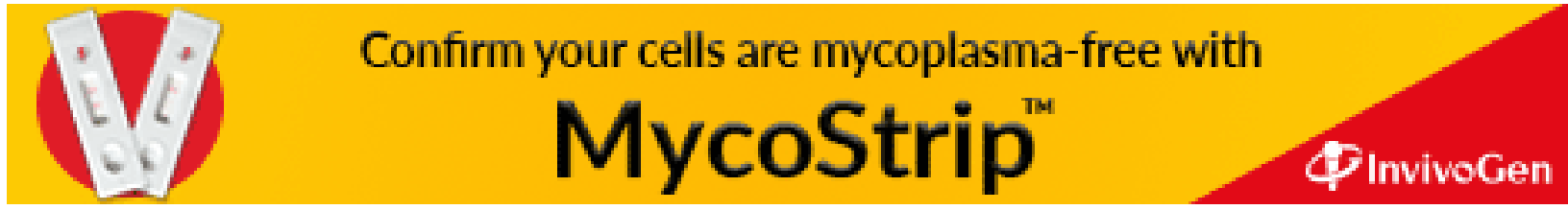

